\title{
Boltzman Equation Analysis of Position and Time Dependent Velocity Distribution of an Electron Swarm
}

\author{
Member Sadanojo Nakajima (Tokushima University) \\ Nom-member Toshihiko Okamoto (Tokushima University) \\ Member Nobuaki Ikuta (Tokushima University)
}

By directly solving the Boltzmann equation in the path integral form, the position and time dependent velocity distribution of electrons $f(\boldsymbol{x}, \boldsymbol{v}, t)$ is accurately calculated perhaps for the first time. In this method, the position and time resolved transport quantities $z(x, t)$ which give clear insight for the inner structure of an electron swarm are easily calculated. The calculation of the transport coefficients in definitions for each type of experiments such as PT, SST, and of arrival time spectra are also possible. The theory and procedures of this analysis and the preliminary results calculated in the ramp model gas of Reid within bounded space are presented with discussion.

Keywords : Position and time dependent velocity distribution, Time of flight distribution, Boltzmann equation analysis, Path integral form, Transport coefficients, Electron swarm, Boundary condition, Non-conservative

\section{Introduction}

In order to observe exact behaviors of spatiotemporal development of an electron swarm in gas under electric field, it is necessary to establish a numerical procedure to calculate the position and time dependent velocity distribution $f(r, v, t)$. Such phase space analysis has been possible even under given initial and boundary conditions, if the MonteCarlo simulation is executed. However, the considerably long computer time and the statistical fluctuation in the results have refused the use of it. The Boltzmann equation analysis, on the other hand, have not been successfully used for the phase space analysis. Kitamori, et al. ${ }^{(1)(2)}$ calculated the temporal development of electron avalanches in neon and argon gas by using the spatial Fourier expansion of Tagashira et al..$^{(3)(4)}$, and by developing the method of direct estimation of moment in real space. Very recently, Drallos and Wadehra ${ }^{(5)(6)}$ presented a direct method to analyse the transient velocity distribution $f(\boldsymbol{v}, t)$ quite independently with present authors ${ }^{(7)}$, though the procedures are similar to each other. But these methods still remain to be the analysis for $f(v, t)$ but not for the phase space distribution $f(\boldsymbol{r}, \boldsymbol{v}, t)$.

On the other hand, Kumar ${ }^{(8)}$ formally solved the Boltzmann equation for the phase space distribution written in a series of terms $f^{k}(v)(-D)^{k} n(r, t)$, but the practical solution has not be shown yet. We developed a calculation procedures to obtain the time of flight distribution $f(\boldsymbol{x}, \boldsymbol{v}, t)$. It is an extension of our previous method ${ }^{(7)}$ to the phase space analysis solving the path integral form Boltzmann equation in a time sequential manner. The procedure in which is different from that of Skullerud ${ }^{(9)}$ in spite of that the same formula is used. In this paper, the theory and procedures of calculation in this method are written in Chap. 2 and in Chap. 3 respectively, and some preliminary results of calculation 
are given in Chap. 4 with discussion relevant to the electron transport property.

\section{Theory}

The Boltzmann equation is usually written as

$$
\left(\frac{\partial}{\partial t}+v \nabla_{r}+\frac{F}{m} \nabla_{v}\right) f(\boldsymbol{r}, \boldsymbol{v}, t)=\left(\frac{\partial f}{\partial t}\right)_{\mathrm{col} 1}
$$

and the collision term of which is given as the balance between the source term $\phi_{s}(r, v, t)$ and the loss term $\psi_{c}(r, v, t)$.

$$
\left(\frac{\partial f}{\partial t}\right)_{\text {coll }}=\psi_{s}(\boldsymbol{r}, \boldsymbol{v}, t)-\phi_{c}(\boldsymbol{r}, \boldsymbol{v}, t)
$$

where

$$
\begin{aligned}
\psi_{s}(\boldsymbol{r}, \boldsymbol{v}, t) & =\left(\frac{\partial f}{\partial t}\right)_{\mathrm{in}} \\
& =\iiint S\left(\boldsymbol{r}, \boldsymbol{v}^{\prime} \rightarrow v, t\right) \phi_{c}\left(\boldsymbol{r}, \boldsymbol{v}^{\prime}, t\right) d \boldsymbol{v}^{\prime} \\
& =\iiint \Omega\left(\boldsymbol{r}, \boldsymbol{v}^{\prime} \rightarrow v, t\right) f\left(\boldsymbol{r}, \boldsymbol{v}^{\prime}, t\right) d \boldsymbol{v}^{\prime}
\end{aligned}
$$

and

$$
\begin{aligned}
\phi_{c}(\boldsymbol{r}, \dot{v}, t) & =\left(\frac{\partial f}{\partial t}\right)_{\text {out }} \\
& =\nu_{T}(\boldsymbol{r}, \boldsymbol{v}, t) f(\boldsymbol{r}, \boldsymbol{v}, t)
\end{aligned}
$$

Here, both of $\phi_{s}(r, v, t)$ and $\psi_{c}(r, v, t)$ are defined as the re-starting (incoming) rate and the colliding (outgoing) rate, respectively, not normalized by total collision frequency $\nu_{T}(r, v, t)$ as those in our previous paper ${ }^{(10)}$. $\quad S\left(\boldsymbol{r}, \boldsymbol{v}^{\prime} \rightarrow \boldsymbol{v}, t\right)$ is the velocity transfer function to the region $v d v$ due to a collision at $v^{\prime} d v^{\prime}$, and $\Omega\left(r, v^{\prime} \rightarrow v, t\right)$ is the collision operator giving the incoming rate into $v d v$ for an electron through collisions at $v^{\prime} d v^{\prime}$ as is usually defined.

Without collisions, the electron density distribution $f(r, v, t)$ along a flight path does not change, and the Boltzmann equation for electrons on the path is written using the total derivative $d f / d t^{(9)}$ as

$$
\left[\frac{d}{d t}+\nu_{T}(\boldsymbol{r}, \boldsymbol{v}, t)\right] f(\boldsymbol{r}, \boldsymbol{v}, t)=\phi_{s}(\boldsymbol{r}, \boldsymbol{v}, t)
$$

and the Boltzmann equation of the path integral form is derived from Eq. ( 5 ) as below.

$$
\begin{aligned}
f(\boldsymbol{r}, \boldsymbol{v}, t)= & f\left[\tilde{\boldsymbol{r}}\left(t_{0} ; \boldsymbol{r}, \boldsymbol{v}, t\right), \tilde{\boldsymbol{v}}\left(t_{0} ; \boldsymbol{r}, \boldsymbol{v}, t\right), t_{0}\right] \\
& \times \exp \left[-\int_{0}^{t} \nu_{T}\{\tilde{\boldsymbol{r}}(\tau ; \boldsymbol{r}, \boldsymbol{v}, t)\right. \\
& \tilde{\boldsymbol{v}}(\tau ; \boldsymbol{r}, \boldsymbol{v}, t), \tau\} d \tau] \\
& +\int_{t_{0}}^{t} d t_{1} \psi_{\mathrm{s}}\left[\tilde{\boldsymbol{r}}\left(t_{1} ; \boldsymbol{r}, \boldsymbol{v}, t\right)\right. \\
& \left.\tilde{\boldsymbol{v}}\left(t_{1} ; \boldsymbol{r}, \boldsymbol{v}, t\right), t_{1}\right] \\
& \times \exp \left[-\int_{t_{1}}^{t} \nu_{T}\{\tilde{\boldsymbol{r}}(\tau ; \boldsymbol{r}, \boldsymbol{v}, t)\right. \\
& \tilde{\boldsymbol{v}}(\tau ; \boldsymbol{r}, \boldsymbol{v}, t), \tau\} d \tau] \cdots \cdots \cdots(6)
\end{aligned}
$$

Here, the position and the velocity at $t_{1}$ along the path which passes $r$ and $v$ at $t$ is

$$
\boldsymbol{r}\left(t_{1}\right)=\tilde{\boldsymbol{r}}\left(t_{1} ; \boldsymbol{r}, \boldsymbol{v}, t\right), \quad \boldsymbol{v}\left(t_{1}\right)=\tilde{\boldsymbol{v}}\left(t_{1} ; \boldsymbol{r}, \boldsymbol{v}, t\right) .
$$

We assume that the electrons collide with gas atoms isotropically in the laboratory frame, and $\psi_{s}(\boldsymbol{r}, v, t)$ is always isotropic. The number of outgoing electrons due to a kind of collision $k$ from the velocity region $d v^{\prime}$ at $v^{\prime}$ and those incoming to $d v$ at $v$ in unit time through conservative collisions have to be the same as written below.

$$
\phi_{s k}(\boldsymbol{r}, v, t) J(v) d v=\phi_{c 0 k}\left(\boldsymbol{r}, v^{\prime}, t\right) J\left(v^{\prime}\right) d v^{\prime}
$$

$$
\psi_{s k}(\boldsymbol{r}, v, t)=\phi_{c 0 k}\left(\boldsymbol{r}, v^{\prime}, t\right) \frac{J\left(v^{\prime}\right)}{J(v)} \frac{d v^{\prime}}{d v}
$$

where $J\left(v^{\prime}\right)$ and $J(v)$ are the Jacobian of the velocity region $d v^{\prime}$ at $v^{\prime}$ and $d v$ at $v$, respectively, and $\psi_{c 0}$ is the isotropic component of $\psi_{c}$.

\section{Calculation procedures}

For electrons of mass $m$, the source term $\psi_{s}(\boldsymbol{r}, \boldsymbol{v}$, $t)$ in the elastic collision with cold gas atoms of mass $M$ is written as

$$
\begin{aligned}
\phi_{s e l}(r, v, t)= & \phi_{c 0 e l}\left(r, \frac{v}{(1-2 m / M)^{1 / 2}}, t\right) \\
& \times \frac{1}{(1-2 m / M)^{3 / 2}} \cdots \cdots \cdots \cdots
\end{aligned}
$$

and that in the excitation collision losing energy $\varepsilon_{e x}$ is written as

$$
\begin{aligned}
\phi_{s e x}(\boldsymbol{r}, v, t)= & \psi_{c 0 e x}\left[\boldsymbol{r},\left(v^{2}+\boldsymbol{V}_{1}^{2} \varepsilon_{e x}\right)^{1 / 2}, t\right] \\
& \times\left(v^{2}+\boldsymbol{V}_{1}^{2} \varepsilon_{e x}\right)^{1 / 2} / v \quad \cdots \cdots
\end{aligned}
$$

For the ionization collision, sharing the residual energy $\left(\varepsilon-\varepsilon_{i}\right)$ with the ratio $\Delta /(1-\Delta)$ to the two electrons scattered and ejected, 


$$
\begin{aligned}
& \psi_{s i_{1}}(\boldsymbol{r}, v, t)=\psi_{c 0 i}\left[\boldsymbol{r},\left(v^{2} / \Delta+\boldsymbol{V}_{1}^{2} \varepsilon_{i}\right)^{1 / 2}, t\right] \\
& \times\left[\left(v^{2} / \Delta+V_{1}^{2} \varepsilon_{i}\right)^{1 / 2} / \Delta v\right] \\
& \left.\psi_{s i_{2}}(\boldsymbol{r}, v, t)=\phi_{c 0 i}\left[\boldsymbol{r},\left(v^{2} / 1-\Delta\right)+\boldsymbol{V}_{1}^{2} \varepsilon_{i}\right)^{1 / 2}, t\right] \\
& \left.\times\left[\left(v^{2} / 1-\Delta\right)+V_{1}^{2} \varepsilon_{i}\right)^{1 / 2} /(1-\Delta) v\right]
\end{aligned}
$$

where

$$
\left.\begin{array}{l}
V_{1} \varepsilon^{1 / 2}=v, \quad(1 / 2) m v^{2}=e \varepsilon \\
\psi_{s i}=\phi_{s i_{1}}+\psi_{s i_{2}}
\end{array}\right\}
$$

In the calculation of $\phi_{s}(\boldsymbol{r}, v, t)$ at lattice points from $\psi_{c 0}\left(\boldsymbol{r}, v^{\prime}, t\right)$ through a collision at $v^{\prime}$, the values of $v^{\prime}$ are not at the lattice points, in general. Then, the second order interpolation technique of Lagrange is adopted to obtain $\psi_{c 0}\left(v^{\prime}\right)$ from $\psi_{c 0}\left(v_{j}^{\prime}\right)$ at lattice points $v_{j}^{\prime}$ as

$$
\psi_{c 0}\left(v^{\prime}\right)=\sum_{j=m-1}^{m+1} \beta_{j}\left(v^{\prime}\right) \phi_{c}\left(v_{j}^{\prime}\right)
$$

where,

$$
\beta_{j}\left(v^{\prime}\right)=\prod_{\substack{s=m-1 \\ s \neq j}}^{m+1} \frac{v^{\prime}-v_{s}^{\prime}}{v_{j}^{\prime}-v_{s}^{\prime}}
$$

and $v_{m}{ }^{\prime}$ is the nearest lattice point of $v^{\prime}$.

In the practical calculation of the velocity distribution, $f(\boldsymbol{x}, \boldsymbol{v}, t)$ are written as $f(\boldsymbol{x}, v, \theta, t)$ assum. ing the symmetry in azimuthal angle. In the calculation of lattice values of $f(\boldsymbol{x}, v, \theta, t)$ from the values $\Delta t$ before, the values of $x(t-\Delta t), v(t-\Delta t)$ and $\theta(t$ $-\Delta t)$ are generally not on the lattices. Then, the value of $f(x(t-\Delta t), \theta(t-\Delta t), t-\Delta t\}$ is calculated from the lattice values nearby, by using similar procedures at collision.

$$
\begin{aligned}
f(x, v, \theta, t-\Delta t) \\
=\sum_{i=l-1}^{l+1} \sum_{j=m-1}^{m+1} \sum_{k=n-1}^{n+1} \alpha_{i}(\boldsymbol{x}) \beta_{j}(v) \gamma_{k}(\theta) \\
\quad \times f\left(\boldsymbol{x}_{i}, v_{j}, \theta_{k}, t-\Delta t\right) \cdots \cdots \cdots \cdots \cdots
\end{aligned}
$$

where,

$$
\left.\begin{array}{l}
a_{i}(\boldsymbol{x})=\prod_{\substack{s=t-1 \\
s \neq i}}^{i+1} \frac{\boldsymbol{x}-\boldsymbol{x}_{s}}{\boldsymbol{x}_{i}-\boldsymbol{x}_{s}} \\
\beta_{j}(\boldsymbol{v})=\prod_{\substack{s=m-1 \\
s \neq j}}^{m+1} \frac{v-v_{s}}{v_{j}-v_{s}} \\
\gamma_{k}(\theta)=\prod_{\substack{s=n-1 \\
s \neq k}}^{n+1} \frac{\theta-\theta_{s}}{\theta_{\boldsymbol{k}}-\theta_{s}}
\end{array}\right\}
$$

and $\boldsymbol{x}_{l}, v_{m}$ and $\theta_{n}$ are the nearest lattice points.

Here, writing in general forms again, the calculation of $f(\boldsymbol{r}, \boldsymbol{v}, t)$ from $f(\boldsymbol{r}, \boldsymbol{v}, t-\Delta t)$ is carried out by adopting a "double half collision method" due to it's better accuracy, where the expression $f(\boldsymbol{r}, \boldsymbol{v}, t$ $-\Delta t)$ implies $f(\tilde{r}(t-\Delta t ; \boldsymbol{r}, \boldsymbol{v}, t), \tilde{\boldsymbol{v}}(t-\Delta t: \boldsymbol{r}, \boldsymbol{v}, t)$, t).

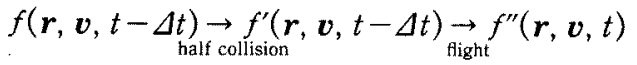

$$
\begin{aligned}
& \underset{\text { half collision }}{\rightarrow} f(\boldsymbol{r}, \boldsymbol{v}, t) \text {. }
\end{aligned}
$$

Here,

$$
\begin{aligned}
f^{\prime}(\boldsymbol{r}, \boldsymbol{v}, t-\Delta t)= & f(\boldsymbol{r}, \boldsymbol{v}, t-\Delta t) \\
& -\sum_{K} \phi_{c K}(\boldsymbol{r}, \boldsymbol{v}, t-\Delta t)(\Delta t / 2) \\
& +\sum_{K} \phi_{s K}(\boldsymbol{r}, \boldsymbol{v}, t-\Delta t)(\Delta t / 2) \\
f^{\prime \prime}(\boldsymbol{r}, \boldsymbol{v}, t)= & f^{\prime}\{\tilde{\boldsymbol{r}}(t), \tilde{\boldsymbol{v}}(t), t\} \\
f(\boldsymbol{r}, \boldsymbol{v}, t)= & f^{\prime \prime}(\boldsymbol{r}, \boldsymbol{v}, t) \\
& -\sum_{K} \phi_{c K}(\boldsymbol{r}, \boldsymbol{v}, t)(\Delta t / 2) \\
& +\sum_{K} \phi_{s K}(\boldsymbol{r}, \boldsymbol{v}, t)(\Delta t / 2) . \cdots(19)
\end{aligned}
$$

It is noted that the distribution $f(r, v, t)$ is easily calculated without using partial differential equations even under the existence of magnetic field only if $\tilde{\boldsymbol{r}}(t)$ and $\tilde{\boldsymbol{v}}(t)$ are given as

$$
\left.\begin{array}{l}
\frac{d \tilde{\boldsymbol{r}}(t)}{d t}=\boldsymbol{v}(t) \\
\frac{d \tilde{\boldsymbol{v}}(t)}{d t}=e[E(\tilde{\boldsymbol{r}}, t)+\tilde{\boldsymbol{v}}(t) \times B(\tilde{\boldsymbol{r}}, t)]
\end{array}\right\}
$$

\section{Results and discussion}

\subsection{Conditions of calculation}

A series of calculation for the time of flight distribution $f(\boldsymbol{x}, \boldsymbol{v}, t)$ and for related transport quantities are carried out in the ramp model gas of Reid ${ }^{(11)}$, the cross sections of which are as below.

Momentum transfer cross section:

$$
q_{m}=6.0 \mathrm{~A}^{2}
$$

Excitation cross section :

$$
\begin{aligned}
q_{e x} & =0 & & \varepsilon \leqq 0.2 \mathrm{eV} \\
& =10(\varepsilon-0.2) \mathrm{A}^{2} & & \varepsilon>0.2 \mathrm{eV}
\end{aligned}
$$

The isotropic scattering of electrons with cold gas of density $N=10^{16} \mathrm{~cm}^{-3}$ is assumed. For the aim to analyse the electron behaviors in practical devices, we adopted a limited position space by perfectly absorptive two plane electrodes at $x=0$ and mainly at $\boldsymbol{x}=3 \mathrm{~cm}$. The initial distributions given here are Gaussian in position space centered at $x=1 \mathrm{~cm}$ and Maxwellian in velocity space, 


$$
\begin{aligned}
f_{0}(\boldsymbol{x}, v, \theta, t)= & \frac{1}{\left(2 \pi \sigma_{0}^{2}\right)^{1 / 2}} \exp \left[-\frac{\left(\boldsymbol{x}-\boldsymbol{x}_{0}\right)^{2}}{2 \sigma_{0}^{2}}\right] \\
& \times \frac{1}{\left(2 \pi e \varepsilon_{0}^{2}\right)^{3 / 2}} \exp \left[-\frac{m v^{2}}{2 e \varepsilon_{0}}\right]
\end{aligned}
$$

where $x_{0}=1 \mathrm{~cm}, \sigma_{0}=0.2 \mathrm{~cm}, \varepsilon_{0}=0.2 \mathrm{eV}$, and the initial number of electrons integrated over both of position and velocity spaces is taken to be unity.

In order to reduce the calculation error, the time interval $\Delta t$ of $0.1 \mathrm{~ns}$ is adopted as the relation $\nu_{T}(v$, t) $\Delta t<0.1$ is held. The divisions $\Delta x: 0.05 \mathrm{~cm}$ in position space and $\Delta v: 0.02 \mathrm{eV}^{1 / 2}$ in velocity space are used in relation to $\Delta t$. Prior to solve the Boltzmann equation, an accuracy examination for the interpolation technique was executed in a collision free condition. The results of which showed a high accuracy in comparison with theoretical values, proving the reliability of this procedure.

\subsection{Variation of $F_{l}(\boldsymbol{x}, v, t)$}

Calculated velocity distribution $f(x, v, t)$ through
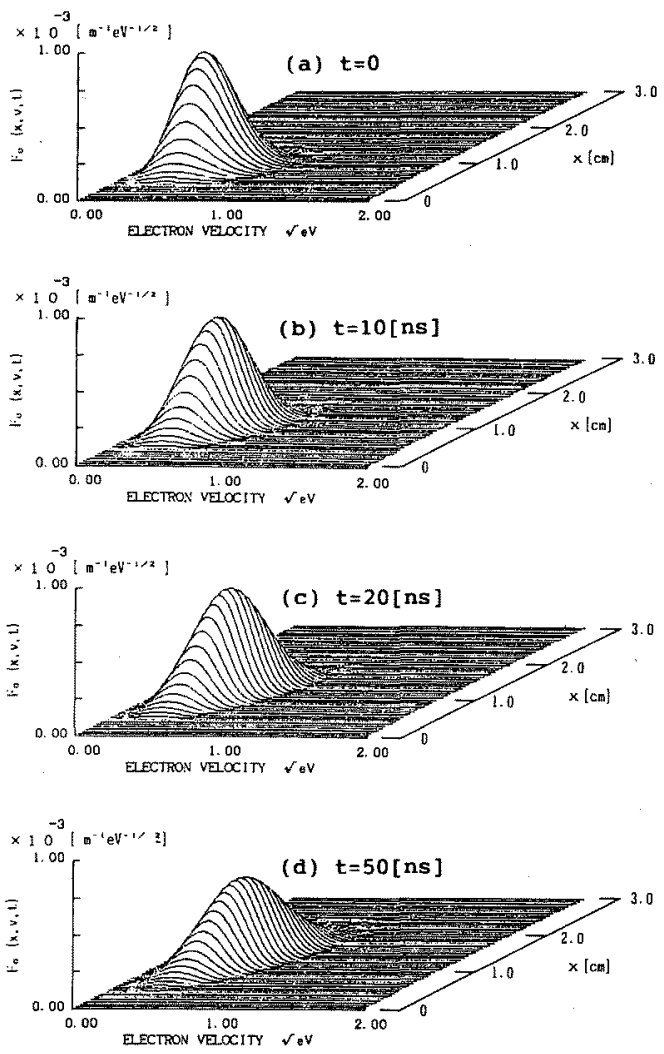

Fig. 1. Variation of $F_{0}(x, v, t)$ at $12 \mathrm{Td}$. collisions with the model gas are conveniently shown in a series of Legendre polynomials, $F_{0}, F_{1}$ and $F_{2}$, exactly deduced from it. In Fig. I (a ) (d), the number distributions $F_{0}(x, v, t)$ calculated under the reduced field $E / N$ of $12 \mathrm{Td}$ at time $0,10,20$
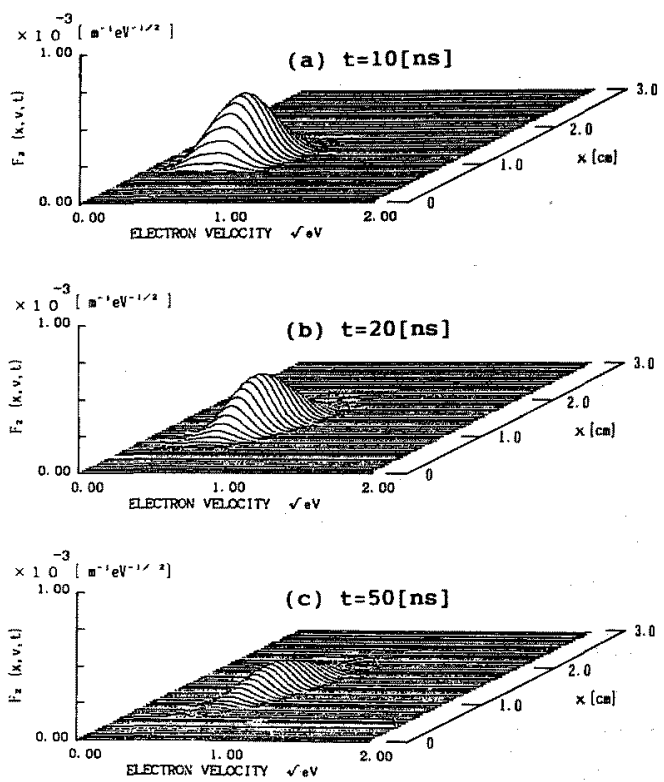

Fig. 2. Variation of $F_{1}(x, v, t)$ at $12 \mathrm{Td}$.
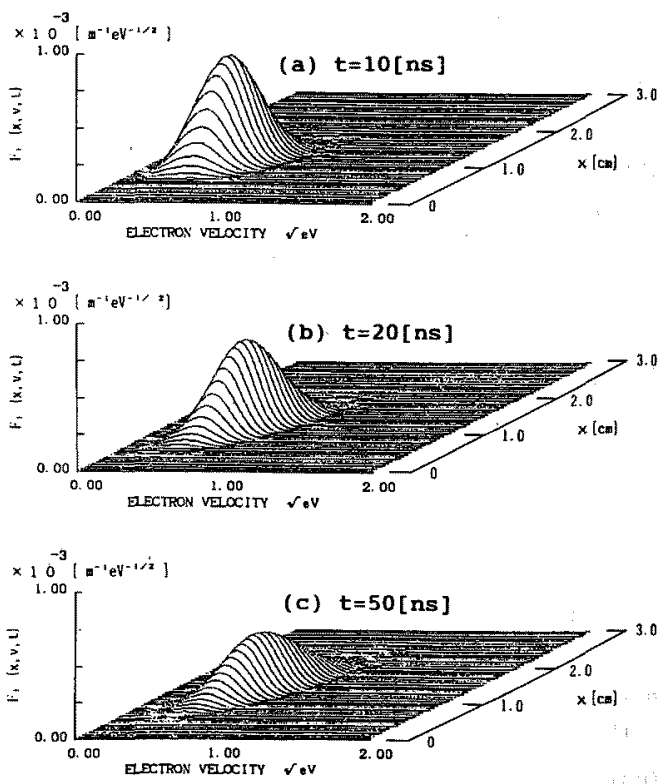

Fig. 3. Variation of $F_{2}(x, v, t)$ at $12 \mathrm{Td}$. 


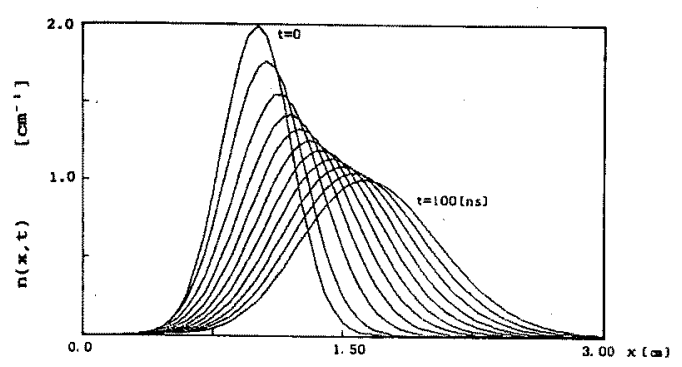

Fig. 4. Temporal variation of $n(x, t)$ at $12 \mathrm{Td}$.

and $50 \mathrm{~ns}$ are shown on the $x-v$ plane ( $x$ in slanted axis and $v$ in abscissa). The time 10,20 and $50 \mathrm{~ns}$ are chosen to show the situation that the electrons are just being accelerated, being decelerated and are almost in equilibrium at each period, respectively. The situation being highly accelerated in the early stage is caused by the long flight of electrons started with low energy, i. e. with low collision frequency. Note here that both of $F_{1}(x, v, t)$ (Fig. 2) and $F_{2}(x$, $v, t$ ) (Fig. 3) are the largest at $10 \mathrm{~ns}$. Since the ordinate scales for all of $F_{0}, F_{1}$ and $F_{2}$ are the same, and the values of $F_{1}$ and $F_{2}$ directly express the anisotropy.

Concerning the contours of $F_{0}(\boldsymbol{x}, v, t)$, the shapes in $x-v$ plane for an electron swarm started from low mean energy may be observed slim along a slanted axis, because the spatial development of electrons simply brings the energy gain. As the time elapses, however, it will gradually be thick, and approaches the equilibrium distribution with less dependence on the position $x$ (cf. Fig. 5).

The time dependent density distribution of $n(x, t)$ are exactly obtained by integrating $f(x, v, t)$ in velocity space as are seen in Fig. 4.

$$
\begin{aligned}
n(\boldsymbol{x}, t) & =\int_{0}^{\pi} \int_{0}^{\infty} f(\boldsymbol{x}, v, \theta, t) 2 \pi v^{2} d v \sin \theta d \theta \\
& =\int_{0}^{\infty} F_{0}(\boldsymbol{x}, v, t) d v \quad \cdots \cdots \cdots \cdots \cdots(22)
\end{aligned}
$$

The shapes of curves of $n(x, t)$ are almost symmetric. This may be caused by the collision frequency characteristics given here which increases with the velocity increase. However, the shapes $n(x, t)$ obtained by a Monte-Carlo simulation under a constant collision frequency condition have markedly extended front end especially in the early stage. In the present calculation, in contrast, the suppression of the front extension due to the increasing collision frequency characteristics with energy have brought the almost symmetric shapes of $n(x, t)$. In such conditions, of course, the longitudinal diffusion coefficient $D_{L}$ appears smaller than that in the transverse direction $D_{T}$.

\subsection{Variation of transport quantities}

Among the calculated results, the transport quantities $z(x, t)$ expressed as the mean values for $n(x$, $t)$ are shown in Fig. 5.

$$
z(x, t)=\frac{\iiint z f(x, v, t) d v}{\iiint f(x, v, t) d v} \cdots \cdots \cdots \cdots \cdots(23)
$$

where the span of each curve implies the length of the distribution $n(x, t)$ limited by $1 \%$ of $n_{\max }(x, t)$. The thick lines with the label $t=0$ give the initial values $z\left(x, t_{0}\right)$ within the limited lengths of $n\left(x, t_{0}\right)$.

All the quantities $z(x, t)$ calculated here rapidly increase in the front side almost linearly, the gradients of which, however, commonly decrease after that. Of course, the fast rise in the front side of each quantity is caused by the low collision frequency characteristics of electrons started from the low energy region.

Fig. 5 (a) shows the time variation of the local drift velocity $W_{v}(x, t)$ :

$$
W_{v}(x, t)=\frac{1}{3} \int_{0}^{\infty} v F_{1}(x, v, t) d v
$$

Within $0 \sim 10 \mathrm{~ns}$, the axial gradient of $W_{v}(x, t)$ rapidly increases to high values. After taking a maximum, however, it decreases in relatively slow pace. Near the anode in Fig. 5(a), a steep increase of $W_{v}(\boldsymbol{x}, t)$ is observed. This is of course caused by the electron absorption at the anode, giving the reduction of backward directed electrons.

The spatial integration of $W_{v}(x, t)$, that is, the time dependent drift velocity $W_{v}(t)$ is obtained as

$$
\begin{aligned}
W_{v}(t) & =\int_{0}^{L} W_{v}(x, t) n(x, t) d x \int_{0}^{L} n(x, t) d \boldsymbol{x} \\
& =\frac{1}{n(t)} \int_{0}^{L} W_{v}(\boldsymbol{x}, t) n(\boldsymbol{x}, t) d x \cdots(25)
\end{aligned}
$$

and takes a typical time behavior having a overshoot similar to $W_{r}(t)$ shown in Fig. 6(a). Such a phenomenon can not be obtained by the analysis using the momentum and energy relaxation times simply adopted by Makabe, et al. ${ }^{(12)}$ Though the 


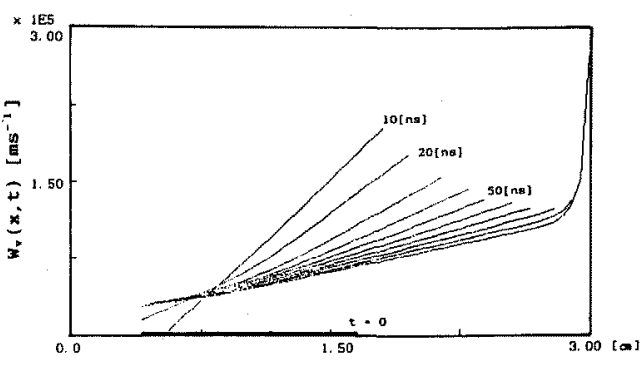

(a) Drift velocity $W_{*}(x, t)\left[\mathrm{ms}^{-1}\right]$
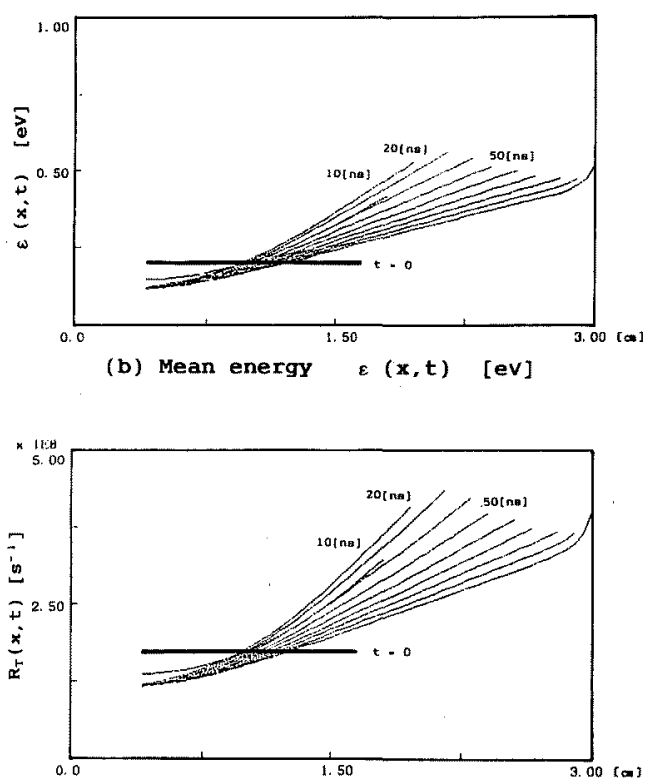

(c) Collision frequency $R_{T}(x, t)\left[s^{-1}\right]$

Fig. 5. Temporal variation of position and time dependent quantities $z(x, t)$ normal. ized with $n(x, t)$ from $0 \sim 100$ ns at $12 \mathrm{Td}$.

calculation by using the relaxation times has a large advantage of simplicity, but cannot respond in detail to the local and time dependent velocity distributions since the momentum and or energy

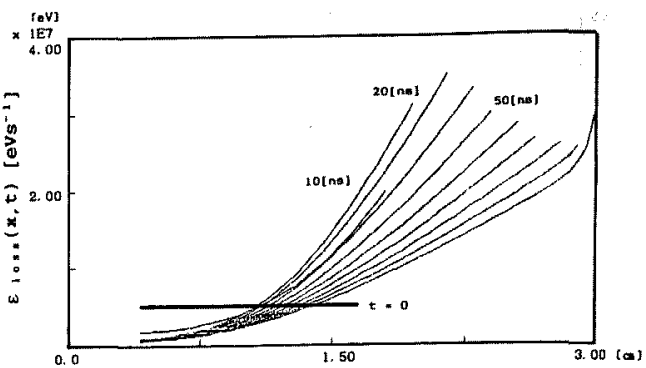

(d) Energy loss rate(total)

$\varepsilon \ldots, \ldots(x, t)\left[\mathrm{eVs}^{-1}\right]$

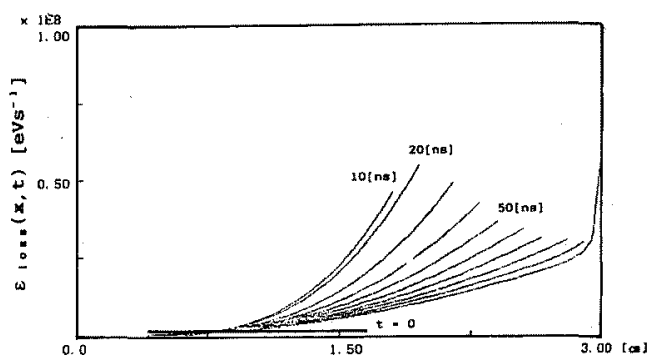

(e) Energy loss rate (parallel)

e $\ldots, \ldots(x, t)\left[\mathrm{eVs}^{-1}\right]$

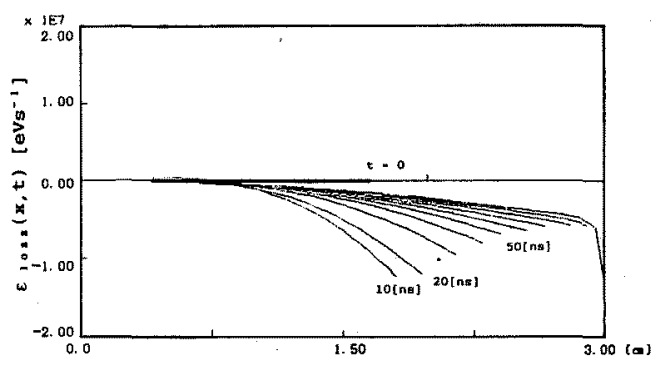

(E) Energy loss rate(perpenndicular) $\varepsilon \ldots \ldots(x, t)\left[\mathrm{eVs}^{-1}\right]$

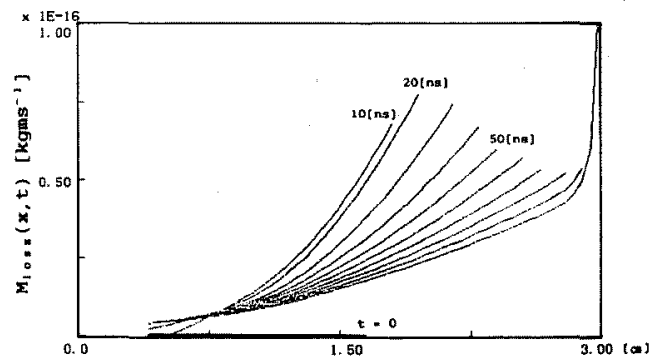

(g) Momentum loss rate(total) $M_{10 \ldots}(x, t)\left[\right.$ kgms $\left.^{-1}\right]$

relaxation times are macroscopic constants.

The variation of local mean energy $\varepsilon(x, t)$ is shown in Fig. 5(b), where similar but slower initial increase $(0 \sim 30 \mathrm{~ns})$ and slower decrease after the 
maximum are observed compared to those of $W_{v}(x$, $t$ ). Near the anode, $\varepsilon(x, t)$ also increases due to the disappearance of electrons by the anode absorption. The total mean energy $\varepsilon(t)$ :

$$
\varepsilon(t)=\frac{1}{n(t)} \int_{0}^{L} \varepsilon(x, t) n(x, t) d x
$$

may change more slowly and take a smaller overshoot due to the decrease of $\varepsilon(x, t)$ at the tail side. Corresponding to $\varepsilon(x, t)$, the local collision frequency $R_{T}(x, t)$ curves shown in Fig. 5(c) are similar but have steeper gradients corresponding to the increasing characteristics of the collision frequency with energy. The variation of the total collision frequency $R_{T}(t)$,

$$
R_{T}(t)=\frac{1}{n(t)} \int_{0}^{L} R_{T}(x, t) n(x, t) d x \quad \cdots(27)
$$

may be larger than $\varepsilon(t)$ due to the same reason.

The curves in Fig. $5(\mathrm{~d})$ show the local energy loss rate $\varepsilon_{\text {loss }}(x, t)$. The variation of $\varepsilon_{\text {loss }}(t)$

$$
\varepsilon_{\mathrm{loss}}(t)=\frac{1}{n(t)} \int_{0}^{L} \varepsilon_{\text {loss }}(x, t) n(x, t) d x \cdots
$$

may be larger than that of $R_{T}(t)$. In Fig. $5(\mathrm{e})$ and $(f)$, the loss rates of parallel and perpendicular components of energies reffered to the field axis are shown. Contrary to the large positive loss rates of parallel energy, the perpendicular energy loss rates appears only negative. This is due to that the perpendicular energy is gained only through collisions. At last, the curves of total momentum loss rates $M_{\text {loss }}(x, t)$ are shown in Fig. $5(\mathrm{~g})$, the time variation of which is similar but faster than that of $\varepsilon_{\text {loss }}(x, t)$.

It is noted here that these space time resolved quantities above shown are able to frankly exhibit the time resolved inner structure of an electron swarm corresponding to the local velocity distributions and the collision frequency characteristics.

Throughout the results shown in Fig. 5, it is apparent that the boundary effects at the anode are remarkable. The cathode effect, on the other hand, does not appear because that the electrons are released apart from the cathode with low velocities.

\subsection{Variation of transport coefficients.}

In the two figures of Fig. 6, the temporal variation of drift velocity $W_{r}(t)$ and of longitudinal diffusion coefficient $D_{L}(t)$ within the limited space of $3 \mathrm{~cm}$ are

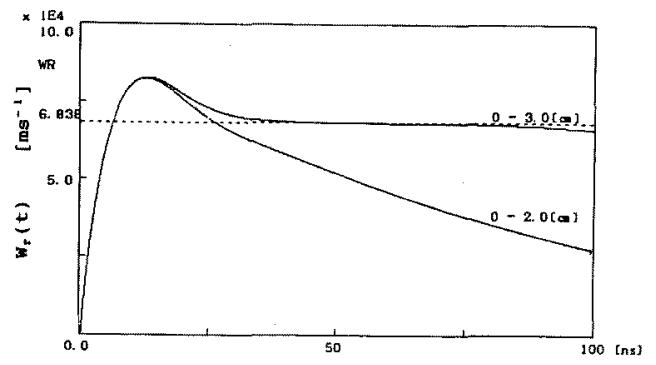

(a) $W_{r}(t)$,

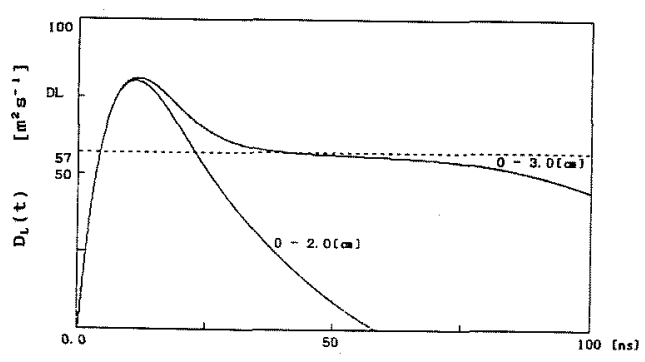

(b) $D_{L}(t)$.

Fig. 6. Temporal variation of drift velocity $W_{\tau}(t)$ and longitudinal diffusion coefficient $D_{L}(t)$ at $12 \mathrm{Td}$.

shown through $0 \sim 100 \mathrm{~ns}$. In the same figures, the curves of $W_{r}(t)$ and $D_{L}(t)$ calculated within shorter position space $(2 \mathrm{~cm})$ are also shown for comparison.

$$
\begin{aligned}
W_{+}(t) & =\frac{d}{d t}\langle\boldsymbol{x}\rangle \\
& =\frac{d}{d t}\left[\int_{0}^{\infty} \int_{0}^{\infty} \boldsymbol{x} F_{0}(x, v, t) d v d \boldsymbol{x}\right]
\end{aligned}
$$

and

$$
\begin{aligned}
D_{L}(t)= & \frac{d}{d t}\left\langle(x-\langle x\rangle)^{2}\right\rangle \\
= & \frac{d}{d t}\left[\int_{0}^{\infty} \int_{0}^{\infty}\left\langle(x-\langle x\rangle)^{2}\right\rangle F_{0}(x, v, t)\right. \\
& \times d v d x] \quad \cdots \cdots \cdots \cdots \cdots \cdots \cdots \cdots \cdots(30)
\end{aligned}
$$

Both the curves of $W_{r}(t)$ in Fig. 6(a) and of $D_{L}(t)$ in Fig. 6(b) take overshoots as are expected from the time variations of $z(x, t)$ observed in Fig. 5 . Although the curves in $3 \mathrm{~cm}$ condition once show tendencies to approach the equilibrium values given by Pitchford ${ }^{(12)}$, Robson ${ }^{(13)}$ and by us ${ }^{(14)}$, shown by dotted lines, but decrease again according to the electron arrivals at the anode. Furthermore, the 
curves in shorter drift length of $2 \mathrm{~cm}$ directly fall down crossing the equilibrium lines due to the arrival and absorption of electrons at the anode from earlier stage. The fall down of these curves from the equilibrium values clearly shows the inadequacy of definitions such as $W_{r}(t)$ and $D_{L}(t)$ to express the electron transport in spatially nonconservative conditions.

\section{Conclusion}

The first attempt to calculate the spatio-temporal development of an electron swarm is carried out by solving the Boltzmann equation in the path integral form. Obtained "time of flight" (TOF) distribution $f(x, v, t)$ is accurate enough, and can provide the swarm parameters fitting to each type of experiments such as "pulsed Townsend" (PT), "steady state Townsend" (SST) and of the "arrival time spectra" (ATS) ${ }^{(15)}$. It can draw the picture of an electron swarm development from start to equilibrium state with arbitrary boundary conditions. The space-time resolved transport quantities $z(x, t)$ deduced from $f(x, v, t)$ give an insight for the inner structure of electron swarm. Another merit of this analysis is the time sequential procedure of calculation, which allow us directly to consider the effects of space charge and of metastables essential in practical situations.

\section{Acknowledgement}

The authors deeply appreciate Mr. A. Harada of our faculty for his aid in preparing this manuscript. (Manuscript received June 1, 1990, reviced Sep. 10, 1990)

\section{References}

(1) K. Kitamori, H. Tagashira \& Y. Sakai : J. Phys. D: Appl. Plys., 11, 283 (1978)

(2) K. Kitamori, H. Tagashira \& Y. Sakai : ibid., : 13, 535 (1980)

(3) H. Tagashira, Y. Sakai \& S. Sakamoto : ibid. : 10, 1051 (1977)

(4) H. Tagashira, T. Taniguchi, K. Kitamori \& Y. Sakai : ibid., . 11, L43 (1978)

(5) P. J. Drallos \& J. M. Wadehra : J. Appl. Phys., 63, 5601 (1988)

(6) P. J. Drallos \& J.M. Wadehra: Phys. Rev.. A40. 1967 (1989)

( 7 ) N. Ikuta. S. Nakajima \& M. Fukutoku: Proc. Jpn. Simp. Plasma Chem., 2, 287 (1989)

(8) K. Kumar : J. Pliys. D: Appl. Plys., 14. 2199 (1981)

(9) H. R. Skullerud \& S. Kuhn : ibid.. 16. 1225 (1983)

(10) N. Ikuta \& Y. Murakami : J. Phys. Soc. Jpn., 56, 115 (1987)

(11) J. D. Reid : Atst. J. Phys.. 32. 231 (1979)

(12) N. Goto \& T. Makabe : J. Phys. D : Appl. Phys., 23, 686 (1990)

(13) L. C. Pitchford, S. V. O'Neil \& J. R. Rumble: Phys. Rev., A23, 294 (1981)

(14) M. Fukutoku \& N. Ikuta: J. Phys. Soc. Jpn., 60 (1990)

(15) K. Kondo \& H. Tagashira : J. Phys. D : Appl. Phys., 23. 1175 (1990)

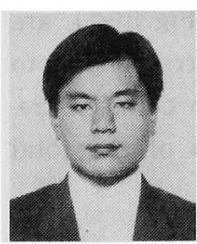

Sadanojo Nakajima (Member)

He was born in Yokkaichi Japan on Sept. 24 in 1960. He received the degrees of $B$. Eng. (1983) and M. Eng. (1985) from Kyoto University, and finished the Dr course in Kyoto University. He began works in Tokushima University in 1988 mainly in the area of fundamental process in gaseous and plasma electronics. He is a member of the IEE Japan and Phys. Soc. Japan.

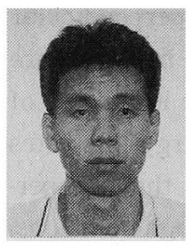

Tadano Ltd.
Toshihiko Okamoto

(Nom-member)

He was born in Kagawa Japan on Sept. 10 in 1966. He received the B. Eng. degree from Tokushima University in 1990, and joined with

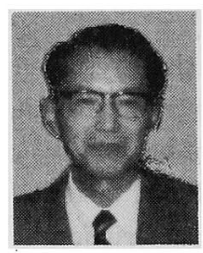

\section{Nobuaki Ikuta (Member)}

He was born in Kohchi Japan on Oct. 22 in 1929. He graduated Tokushima Technical College in 1951. Via a technical high school in Tokushima, he joined Tokushima University in 1964 and is the Professor in Dept. Electronic Engineering. His interest is mainly in the area of fundamental process of discharge phenomena and in gaseous electronics. He received the Dr. Eng. from the University of Tokyo and the Prize of Outstanding Paper from the IEE Japan both in 1972. He is a member of IEE Japan, Phys. Soc. Japan and Japan Soc. of Appl. Phys. 